
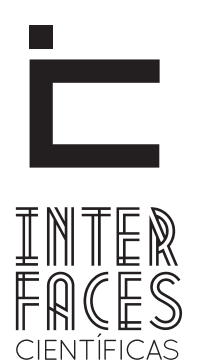

SAÚDE E AMBIENTE

ISSN IMPRESSO 2316-3313

E - ISSN 2316-3798

DOI - 10.17564/2316-3798.2018v6n2p33-42

\title{
CARACTERIZAÇ̃̃O DA MARCHA DO IDOSO COM LOMBALGIA E SUA RELAÇ̃̃O COM RISCO DE QUEDA
}

CHARACTERIZATION OF THE MARCH OF THE ELDERLY WITH LOWER BACK PAIN AND ITS RELATIONSHIP WITH RISK OF FALL

CARACTERIZACIÓN DE LA MARCHA DEL IDOSO CON LUMBALGIA Y SU RELACIÓN CON EL RIESGO DE CAÍDA

Mateus Dias Antunes ${ }^{1}$

Dayele Cruz da Silva ${ }^{3}$

Flavio Bortolozzi ${ }^{5}$
Taynara Fernanda Cardoso Barbosa²

Tânia Maria Gomes da Silva ${ }^{4}$

Sonia Maria Marques Gomes Bertolini ${ }^{6}$

\section{RESUMO}

O envelhecimento é um processo dinâmico e progressivo, caracterizado por alterações morfológicas, bioquímicas e funcionais do organismo. Este se altera progressivamente, tornando-se mais susceptível a acometimentos intrínsecos e extrínsecos, entre eles o risco de quedas. A prevalência de quedas em idosos pode levar a diversas consequências, entre elas a diminuição do entusiasmo e do bem-estar, impactando negativamente a qualidade de vida. Este estudo objetivou caracterizar a marcha do idoso com lombalgia e relacioná-la com o risco de quedas. A amostra foi constituída por 30 mulheres idosas, com idade igual ou superior a 60 anos. Através da análise de passos demarcados no solo foram avaliados o tempo e a velocidade da marcha, o comprimento do passo e da passada, bem como a cadência da mesma. Para avaliação do risco utilizou-se o questionário Fall Risk Score de Dowton. Na avaliação da cadência (112,13 passos/ $\mathrm{min})$, observou-se valores inferiores aos descritos 
pela literatura. Nos resultados obtidos verificou-se que, embora a lombalgia não apresente forte relação com o risco de quedas, leva à limitação física dos idosos e compromete a qualidade de vida.

\section{PALAVRAS-CHAVE}

Dor Lombar. Envelhecimento. Equilíbrio Postural. Promoção da Saúde.

\section{ABSTRACT}

Aging is a dynamic and progressive process, characterized by morphological, biochemical and functional alterations of the organism. It changes progressively, becoming more susceptible to intrinsic and extrinsic disorders, including the risk of falls. The prevalence of falls in the elderly can lead to several consequences, among them the decrease of enthusiasm and well-being, negatively impacting the quality of life. This study aimed to characterize gait of the elderly with low back pain and to relate it to the risk of falls. The sample consisted of 30 elderly women, aged 60 years or older. Through the analysis of demarcated steps in the soil were evaluated the time and the speed of the march,

\section{RESUMEN}

El envejecimiento es un proceso dinámico y progresivo, caracterizado por alteraciones morfológicas, bioquímicas y funcionales del organismo. Este se altera progresivamente, haciéndose más susceptible a acometimientos intrínsecos y extrínsecos, entre ellos el riesgo de caídas. La prevalencia de caídas en ancianos puede llevar a diversas consecuencias, entre ellas la disminución del entusiasmo y del bienestar, impactando negativamente la calidad de vida. Este estudio objetivó caracterizar la marcha del anciano con the length of the step and of the step, as well as the cadence of the same. The Dowton Fall Risk Score questionnaire was used to assess the risk. In the evaluation of cadence (112.13 steps/min), values lower than those described in the literature were observed. In the obtained results, it was verified that, although low back pain does not present a strong relation with the risk of falls, it leads to the physical limitation of the elderly and compromises the quality of life.

\section{KEYWORDS}

Lumbar Pain. Aging. Postural Balance. Health Promotion.

lumbalgia y relacionarla con el riesgo de caídas. La muestra fue constituida por 30 mujeres mayores, de edad igual o superior a 60 años. A través del análisis de pasos demarcados en el suelo se evaluaron el tiempo y la velocidad de la marcha, la longitud del paso y de la pasada, así como la cadencia de la misma. Para la evaluación del riesgo se utilizó el cuestionario Fall Risk Score de Dowton. En la evaluación de la cadencia (112,13 pasos/min), se observaron valores inferiores a los descritos por la literatura. En los resultados ob- 
tenidos se verificó que, aunque la lumbalgia no presente una fuerte relación con el riesgo de caídas, lleva a la limitación física de los ancianos y compromete la calidad de vida.

\section{INTRODUCÇÃO}

Um dos sintomas mais comuns das disfunções da coluna vertebral são as lombalgias. Têm sido evidenciados em diversos estudos que ambos os sexos são afetados (PIRES; SAMULSKI, 2006). As lombalgias representam uma das maiores causas de incapacidades nos indivíduos idosos, pois elas interferem negativamente na qualidade de vida na longevidade (EHRLICH, 2003).

No envelhecimento ocorre um processo dinâmico e progressivo caracterizado por alterações morfológicas, bioquímicas e funcionais, que progressivamente vão se alterando e ficando mais susceptível a acometimentos intrínsecos e extrínsecos que podem levar o ser humano a óbito (PAPALÉO NETTO, 2002).

Há um aumento da população idosa no país e no mundo, resultado de um acréscimo das doenças associadas ao envelhecimento, que são as crônico-degenerativas. Com isso, essas patologias interferem, levando as disfunções em diversos órgãos e funções nos idosos, como os distúrbios do equilíbrio e da postura (ESCUDERO et al., 2001).

Para constatar problemas funcionais associados à locomoção, faz-se necessária a análise da marcha. Diversos estudos revelam que o processo do envelhecimento nos sistemas do nosso corpo promove uma desordem anatômica e funcional que pode acarretar os encurtamentos musculares e diminuição da força, perda de mobilidades articulares e sensoriais que interferem e acometem a mobilidade geral do corpo, expondo a modificações na macha (MACIEL; GUERRA, 2005).

0 equilíbrio é controlado por meio da manutenção do centro de gravidade acima da base de

\section{PALABRAS CLAVE}

Dolor Lumbar. Envejecimiento. Equilibrio Postural. Promoción de la Salud.

sustentação durante as condições dinâmicas e estáticas. Para isso, o corpo deve corresponder essas variações de centro de gravidade de forma involuntária ou voluntária. Para que este processo seja eficaz, ele ocorre por meio do sistema vestibular e visual e somato-sensorial. No envelhecimento, ocorre um aumento da instabilidade do equilíbrio, pois estes sistemas estão alterados e as etapas do controle postural podem estar diminuídas e suprimindo a capacidade compensatória desses sistemas (SÁ; BACHION; MENEZES, 2012).

A queda é caracterizada como um deslocamento não intencional do corpo para um nível inferior à posição inicial, não havendo correção de tempo hábil e é ocasionada por circunstâncias multifatoriais que interferem na estabilidade. Os fatores que levam a queda podem ser separados em fatores intrínsecos, que são aquele que estão relacionados com as alterações fisiológicas consequentes do processo de envelhecimento. Um dos exemplos desses fatores intrínsecos, são das condições patológicas e consumo de medicamentos. Já os fatores extrínsecos estão relacionados com os perigos ambientais, devido às inadequações mobiliárias e arquitetônicas em que maior parte dos idosos estão expostos (GOMES et al., 2014).

Segundo Reis e Flôres (2014) as consequências das quedas podem ser fraturas, traumatismos cranianos e até a morte. Além disso, interferem negativamente na qualidade de vida, provocando sentimentos de medo, desconfiança e fragilidade, sendo diversas vezes caracterizada como o início da degeneração do quadro geral do idoso, pois, 
além de modificar sua mobilidade, prejudica suas atividades sociais e recreativas. Seu custo social torna-se maior quando o idoso tem diminuição da independência e da autonomia, ou necessita de institucionalização (BIAZUS; BALBINOT; WIBERLINGER, 2010). Esse estudo objetivou caracterizar a marcha de idosos com lombalgia e relacioná-la com o risco de quedas.

\section{MÉTODO}

Tratou-se de estudo observacional, com variáveis qualitativas e quantitativas, desenvolvido com um grupo de idosos na cidade de Itambé, no Paraná. A amostra foi constituída de 30 idosos do sexo feminino, com idade igual ou superior a 60 anos. 0 critério de inclusão foi ser idoso diagnosticado com lombalgia há mais de três meses, já os critérios de exclusão consideraram os indivíduos que tiveram doenças inflamatórias e infecciosas da coluna vertebral, fraturas, tumores e vertigem.

Os idosos andaram em uma linha reta demarcada no solo, com distância de 10 metros, cronometrando-se o tempo percorrido. Em relação ao comprimento do passo, do tamanho da passada e da largura foram utilizados papéis do tipo Kraft contínuo, de 10 metros de comprimento e 70 centímetros de largura. Foi colocada na região dos calcâneos uma caneta de demarcação da marca compactor, de cor preta, de acordo com protocolo de Cerny (1983), buscando melhor visualização para análise da mensuração do tamanho do passo e da passada. A análise da cadência, que se refere à quantidade de passos por minuto, foi calculada por regra de três, somando a quantidade de passos total e dividindo por tempo gasto percorrido nos 10 metros e por fim multiplicado por 60 segundos (MASTRANDEA, 2008).

Foi utilizada a Fall Risk Score de Downton para avaliar o risco de queda da população idosa em cinco critérios: quedas anteriores; uso de alguma medicação; presença de algum déficit sensorial; avaliação do estado mental; e avaliação da marcha. A variação de pontuação deste instrumento é de 0 a 11 pontos, e o idoso que é classificado com um maior risco de queda apresenta uma pontuação igual ou superior a três (REIS; ROCHA; DUARTE, 2014).

A Escala Visual Analógica (EVA) foi selecionada para quantificar e verificar a relação da presença de sintomas dolorosos. Desse modo, o método consistiu em aferir numericamente em uma linha reta, graduada de zero a dez, a intensidade da dor, onde zero representa nenhuma dor e dez o máximo de dor (MARTINS et al., 2010).

Foi aplicado o questionário Medical Outcomes Study 36 - Item Short-Form Health Survey (SF-36), traduzido e validado para o português, que avaliou o estado de saúde dos indivíduos. 0 questionário é formado por 36 itens, divididos em oito domínios: capacidade funcional, aspectos físicos, dor física, estado geral de saúde, vitalidade, aspectos sociais, aspectos emocionais e saúde mental. Cada domínio foi avaliado separadamente. As pontuações das questões variam de 0 a 100 pontos, sendo que zero representa um pior estado geral de saúde e cem refere-se a um melhor estado de saúde (LEITE et al., 2012).

Os resultados obtidos foram analisados por meio de estatística descritiva (média, desvio padrão, frequência absoluta e relativa) e inferencial. Foi calculado o coeficiente de correlação de Pearson, com nível de significância de $5 \%$.

Este estudo segue os preceitos da Resolução Ética 466/2012, com aprovação do Comitê de Ética em Pesquisa do Centro Universitário de Maringá - CESUMAR (parecer no 1.359.824; CAAE51658115.9.0000.5539). Todos os participantes receberam explicações sobre a pesquisa e assinaram o Termo de Consentimento Livre e Esclarecido.

\section{RESULTADOS}

Os dados referentes aos parâmetros da marcha dos idosos estão apresentados na Tabela1. 
Tabela 1 - Parâmetros da marcha em idosos com lombalgia

\begin{tabular}{llll}
\hline Parâmetros da Marcha & Média & Desvio padrão & Coeficiente de Variação \\
\hline Tempo $(\mathrm{s})$ & 13,33 & 3,92 & 29,40 \\
Velocidade $(\mathrm{m} / \mathrm{s})$ & 0,83 & 0,28 & 33,73 \\
Comprimento do passo $(\mathrm{cm})$ & 48,03 & 7,79 & 16,21 \\
Comprimento da passada $(\mathrm{cm})$ & 91,60 & 23,61 & 23,69 \\
Largura do passo (cm) & 12,83 & 2,81 & 21,90 \\
Cadência (passos/min) & 112,13 & 48,05 & 42,85 \\
\hline
\end{tabular}

Fonte: Dados da pesquisa.

Em relação aos domínios de qualidade de vida o menor escore foi encontrado na limitação por aspecto físico e o maior na vitalidade (Tabela 2).

Tabela 2 - Escores dos domínios de qualidade de vida de idosos com lombalgia

\begin{tabular}{llll}
\hline Domínios de Qualidade de vida & Média & Desvio padrão & Coeficiente de Variação \\
\hline Capacidade Funcional & 71,16 & 22,42 & 31,50 \\
Limitação por aspecto físico & 55,16 & 42,23 & 76,55 \\
Dor & 59,93 & 29,33 & 48,94 \\
Estado geral de saúde & 66,43 & 14,24 & 21,43 \\
Vitalidade & 74,83 & 10,32 & 13,79 \\
Social & 72,90 & 25,02 & 34,32 \\
Aspecto mental & 70,89 & 20,56 & 29,02 \\
Limitação no aspecto emocional & 59,98 & 40,50 & 67,52 \\
\hline
\end{tabular}


Quando se correlacionou risco de queda e a dor (EVA) dos idosos, bem como a cadência da marcha com a dor, verificou-se uma fraca correlação, não apresentando significância estatística (Figuras 1 e 2). No entanto, verificou-se forte correlação entre a limitação do aspecto físico (SF36) e a dor (Figura 3), o que levaria a uma alteração na qualidade de vida dos indivíduos.

Figura 1 - Correlação entre o Risco de queda e a dor (EVA) em idosos com lombalgia $(r=0,159 ; p>0,05)$

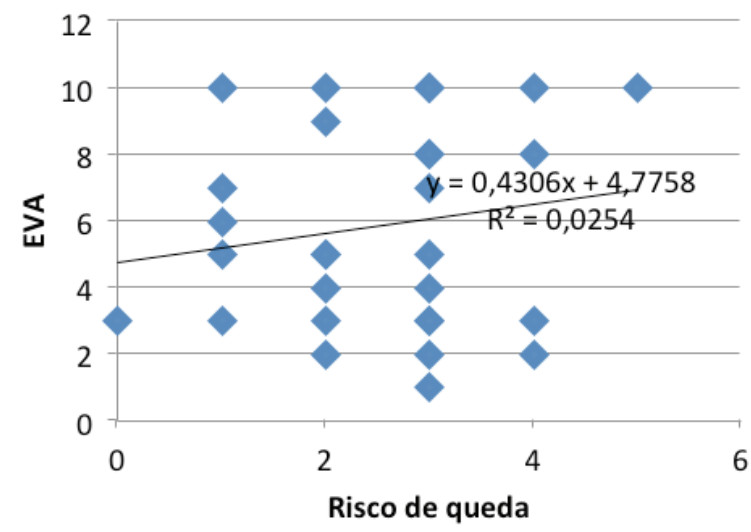

Fonte: Dados de pesquisa.

Figura 2 - Correlação entre a cadência da marcha e a dor (EVA) em idosos com lombalgia $(r=0,217 ; p>0,05)$

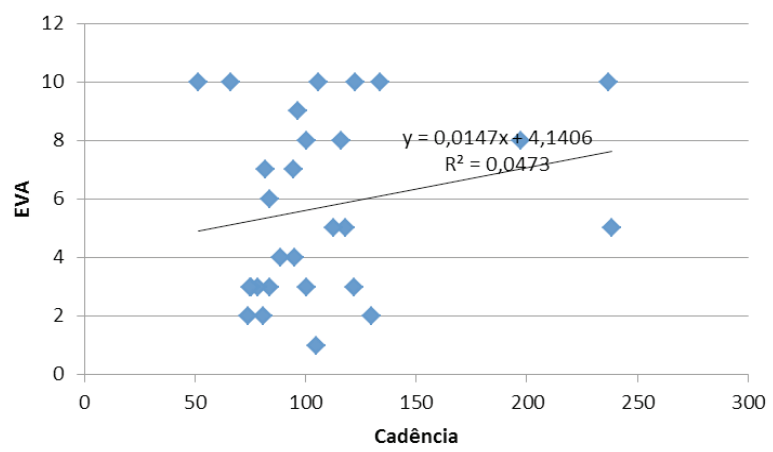

Fonte: Dados de pesquisa.
Figura 3 - Correlação entre os domínios de qualidade de vida limitação do aspecto físico e a dor em idosos com lombalgia $(r=0,698 ; p<0,05)$

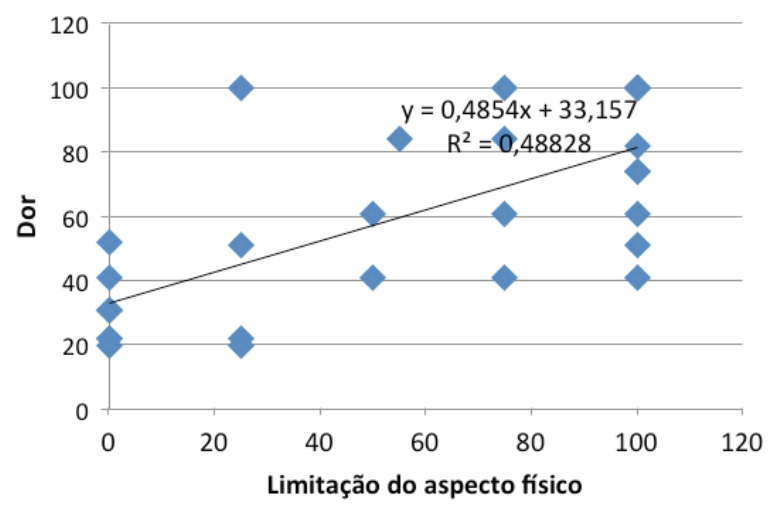

Fonte: Dados de pesquisa.

\section{DISCUSSÃO}

No presente estudo não foi encontrada correlação estatisticamente significativa entre a lombalgia (dor avaliada pela EVA) e o risco de quedas. Entretanto, $70 \%$ das idosas tiveram quedas anteriores e $56 \%$ das idosas apresentaram alto risco de queda.

Chu, Chi e Chiu (2005), encontraram correlação significativa entre queda e a idade mais avançada dos indivíduos. As doenças crônicas, interações farmacológicas ou outras disfunções podem influenciar as alterações fisiológicas do envelhecimento em relação ao controle postural (GAZZOLA et al., 2006).

$\mathrm{Na}$ avaliação da marcha, obteve-se a média de cadência de 112,1 passos/min, comprimento do passo de $48,0 \mathrm{~cm}$ e da passada de $91,6 \mathrm{~cm}$, em uma média de tempo de 13,3 segundos. A caracterização da marcha dos idosos, segundo a literatura, é mais deficitária quando comparada com a faixa etária adulto-jovem. Esse declínio é justificado pelo processo de envelhecimento marcado por diversos eventos biológicos, que ocorrem nos indivíduos com certa progressão e que se relaciona com a prática de atividades e com o nível de exigência destas atividades (PRANKE; TEIXEIRA; MOTA, 2006) 
Oberg, Karsznia e Oberg (1993), constataram um comprimento do passo de $55 \mathrm{~cm}$ em mulheres saudáveis, sedentárias com idade entre 60 a 69 anos. Castro et al. (2000), que estudaram o perfil da marcha de idosas entre 60 e 79 anos, encontraram, na análise do comprimento do passo, valores mais próximos ao do presente estudo $(46 \mathrm{~cm})$.

0 comprimento da passada dos idosos analisados foi de $91,6 \mathrm{~cm}$. Tomando-se como parâmetro os achados de estudos de Murray, Kory e Clarkson (1969), que examinaram 16 idosos, divididos igualmente entre duas faixas etárias - de 60 a 65 e de 63 a 73 anos, mostraram como resultado valores médios de $151 \mathrm{~cm}$ e $136 \mathrm{~cm}$, respectivamente, de comprimento de passada. Entretanto, os escores do presente estudo foram inferiores.

Os valores médios encontrados para cadência foram inferiores (112,13 passos/min) aos obtidos por Castro et al. (2000), constatando 137,4 $\pm 22,64$ passos/min para mulheres de 60 a 79 anos. Estes resultados comparam-se com os do estudo de Murray, Kory e Clarkson (1969), que observaram a média da cadência de 115 passos/min ao examinarem 12 idosos com idade entre 60 e 65 anos.

Acredita-se, considerando que os idosos têm uma diminuição da velocidade da marcha, que isso seja um fator de proteção às quedas e não o seu mecanismo causador (BAIRD; VAN EMMERIK, 2009), ou seja, os idosos não caem devido a caminharem muito lentamente, mas caminham com passos lentos como uma forma de se precaverem de eventuais quedas.

\section{CONCLUSÃO}

0 presente estudo concluiu que embora a dor resultante das lombalgias seja fator limitante à vida do idoso, nos casos analisados não provocou significativas alterações na marcha e nem ocasionou altos índices de quedas, embora esse resultado não deva obscurecer o fato de que toda dor reduz a autonomia do sujeito e compromete sua qualidade de vida.

Desse modo, considera-se importante que novos estudos sejam feitos, a fim de que, cada vez mais, se construam conhecimentos que levem ao fortalecimento da autonomia dos idosos. A interdisciplinaridade, nesse caso, poderá ser uma ferramenta importante, alargando as fronteiras do conhecimento e permitindo uma maior compreensão das questões inerentes ao envelhecer.

\section{AGRADECIMENTOS}

Ao Instituto Cesumar de Ciência, Tecnologia e Inovação (ICETI) e à Coordenação de Aperfeiçoamento de Pessoal de Nível Superior (CAPES) que contribuiu com esta investigação.

\section{REFERÊNCIA}

BAIRD, J.L.; VAN EMMERIK, R.E.A. Young and older adults use different strategies to perform a standing turning task. Clin Biomech, v. 24, n. 10, p. 826-832, 2009.

BIAZUS, M.; BALBINOT, N.; WIBERLINGER, L.M. Avaliação do risco de quedas em idosos. Rev Bras

Ciênc Envelhec Humano, v. 7, n. 1, p. 34-41, 2010.

CASTRO, C.L.N. et al. Estudo da marcha em idosos: resultados preliminares. Acta Fisiátr, v. 7, n. 3, p. 103-107, 2000.

CERNY, K. A clinical method of quantitative gait analysis. Physic Therapy, v. 63, n. 7, p. 1125-1126, 1983.

CHU, L.W.; CHI, I.; CHIU, A.Y. Incidence and predictors of falls in the chinese elderly. Ann Acad Med

Singapore, v. 34, p. 60-72, 2005.

EHRLICH, G. Low back pain. Bull World Health

Organ, v. 81, n. 9, p. 671-676, 2003.

ESCUDERO, S. et al. Incidencia y fatores predictores de inmovilización crónica en ancianos mayores de 75 anos que vivem en la Comunidad. Rev Esp Geriatr

Gerontol, v.36, n.2, p.103-108, 2001. 
GAZZOLA, J.M. et al. Fatores associados ao equilíbrio funcional em idosos com disfunção vestibular crônica. Rev Bras Otorrinolaringol, v. 72, n. 5, p. 683-690, 2006.

GOMES, E.C.C. et al. Fatores associados ao risco de quedas em idosos institucionalizados: uma revisão integrativa. Ciênc Saúde, v. 19, n. 8, p. 3543-3551, 2014.

LEITE, M.T. et al. Qualidade de vida e nível cognitivo de pessoas idosas participantes de grupos de convivência. Rev Bras Geriatr e Gerontol, v. 15, n. 3, p. 481-492, 2012.

MACIEL, A.C.C.; GUERRA, R.O. Prevalência e fatores associados ao déficit de equilíbrio em idosos. Rev

Bras Ciênc Mov, v. 13, n.1, p. 37-44, 2005.

MARTINS, M.R.I. et al. A eficácia da conduta do grupo de postura em pacientes com lombalgia crônica. Rev Dor, v. 11, n. 2, p. 116-121, 2010.

\section{MASTRANDEA, L. Avaliação da marcha em idosas} ativas e sedentárias. Dissertação - Faculdade de Medicina da Universidade de São Paulo, 2008.

MURRAY, M.P.; KORY, R.C.; CLARKSON, B.H. Walking patterns in healthy old men. J Gerontol, v. 24, n. 2, 169-178, 1969.
PAPALÉO NETTO, M. Gerontologia: a velhice e o envelhecimento em visão globalizada. São Paulo: Atheneu, 2002.

OBERG, T.; KARSZNIA, A.; OBERG, K. Basic gait parameters: reference data for normal subjects, 10-79 years of age. J Rehabil Res Dev, v. 30, n. 2, p. 210-231, 1993.

PIRES, F.O.; SAMULSKI, D. Visão interdisciplinar na lombalgia crônica causada por tensão muscular. Rev Bras Ciênc Mov, v. 14, n. 1, p. 13-20, 2006.

PRANKE, G.I.; TEIXEIRA, C.S.; MOTA, C.B. Contribuições biomecânicas ao público da terceira idade. Rev Bras Geriatr Gerontol, v. 9, n. 2, p. 75-91, 2006.

REIS, L.A.; FLÔRES, C.M.R. Relação do risco de quedas e fatores associados em idosos. Rev Baian Enferm, v. 28, n. 1, p. 42-49, 2014.

REIS, L.A.; ROCHA, T.S.; DUARTE, S.F.P. Quedas: risco e fatores associados em idosos institucionalizados. Rev Baian Enferm, v. 28, n. 3, p. 225-234, 2014.

SÁ, A.C.A.M.; BACHION, M.M.; MENEZES, R.L. Exercício físico para prevenção de quedas: ensaio clínico com idosos institucionalizados em Goiânia, Brasil. Ciênc Saúde Coletiva, v. 17, n. 8, p. 21172127, 2012. 
1 Fisioterapeuta; Mestrando em Promoção da Saúde do Centro Universitário de Maringá - UNICESUMAR-PR; Bolsista da Coordenação de Aperfeiçoamento de Pessoal de Nível Superior - CAPES. E-mail: mateus_ antunes03@hotmail.com

2 Acadêmica em Fisioterapia, Centro Universitário de Maringá UNICESUMAR-PR; Bolsista do Programa de Bolsas de Iniciação Científica da UNICESUMAR - PROBIC. E-mail: taynarafernandinha@hotmail.com

3 Acadêmica em Fisioterapia, Centro Universitário de Maringá UNICESUMAR-PR. E-mail: dayele@hotmail.com

4 Historiadora; Doutora em História - UFPR; Docente do Programa de Pós-Graduação em Promoção da Saúde, Centro Universitário de Maringá - UNICESUMAR-PR; Pesquisadora do Instituto Cesumar de Ciência, Tecnologia e Inovação - ICETI. E-mail: tania.gomes@unicesumar.edu.br

5 Matemático e Engenheiro Civil; Doutor em Engenharia de Sistemas Informática pelo Universitè de Technologie de Compiègne, França;

Coordenador do Programa de Pós-Graduação em Gestão do Conhecimento e Professor do Programa de Pós-Graduação em Promoção da Saúde, Centro Universitário de Maringá - UNICESUMAR-PR. E-mail: flavio.bortolozzi@ unicesumar.edu.br

6 Fisioterapeuta; Doutora em Ciências Morfofuncionais - USP;

Coordenadora do Programa de Pós-Graduação em Promoção da Saúde, Centro Universitário de Maringá - UNICESUMAR-PR; Pesquisadora do Instituto Cesumar de Ciência, Tecnologia e Inovação - ICETI. E-mail: sonia. Avaliado em: 22 de Novembro de 2017
Aceito em: 23 de Novembro de 2017 bertolini@unicesumar.edu.br 
